\title{
Polyaniline coated curauá fibres in polyamide- 6 composites: the effect of fibre surface modification on the crystallographic properties
}

\author{
J. R. Araujo ${ }^{1}$, C. B. Adamo ${ }^{2}$, E. Robertis ${ }^{1}$, A. Kuznetsov ${ }^{1}$, \\ B. Archanjo ${ }^{1}$, C. A. Achete $^{1} \&$ M.-A. De Paoli ${ }^{2}$ \\ ${ }^{1}$ Divisão de Metrologia de Materiais, Instituto Nacional de Metrologia, \\ Normalizacão e Qualidade Industrial (INMETRO), Brazil \\ ${ }^{2}$ Institute of Chemistry, University of Campinas (UNICAMP), Brazil
}

\begin{abstract}
Composites have the ability to combine the individual properties of constitutive components, generating a unique material with properties suitable for a particular purpose. This study aims to develop a polymeric reinforced composite material, which is antistatic and light. Polyaniline (PAni) is one of the most studied conductive polymers due to its ability to exist in a large number of intrinsic redox states. The possibility of carrying out PAni synthesis on vegetable fibres and incorporating this in polymeric matrices, such as polyamide- 6 , enables the production of antistatic reinforced materials. In this work, curauá fibres were chemically treated with polyaniline doped with p-toluene sulfonic acid and processed with polyamide- 6 in a twin-screw co-rotating and interpenetrating extruder. The microstructures of the neat curauá fibres and polyaniline coated curauá fibres were characterized by X-ray diffraction (XRD) measurements. Scanning electron microscopy was employed to observe the distribution and morphology of the PAni nanoparticles. The oxidation states of PAni were evaluated by X-ray photoelectron spectroscopy. X-ray diffraction combined with microscopy analyses of PAni synthesized without a substrate, showed crystalline components embedded into amorphous regions in the sample, while the PAni synthesized on the fibres showed a lower degree of crystallinity. The XPS
\end{abstract}


analysis shows that the PAni synthesized on the fibres is in the emeraldine salt form.

Keywords: coating, short-fibre composites, surface treatments, photoelectron spectroscopy, extrusion, degree of crystallinity.

\section{Introduction}

Hybrid materials, such as conducting polymer/polymer composites, are a good way to combine the enhanced properties of the components, namely, the mechanical strength of the reinforced polymeric matrix, and the electrical conductivity of the conducting polymer. Polyamide-6 (PA-6) is an insulating matrix that generates accumulation of static electricity on its surface. The surface coating of an insulating polymer matrix, as PA-6, with a conducting polymer like polyaniline (PAni), may decrease the surface electrical resistance promoting a fast dissipation of a static charge accumulated at the surface [1]. The addition of antistatic agents, however, has a significant effect on the properties of the product, especially mechanical properties [2].

Antistatic materials have very diverse technological applications. Their main purpose is to dissipate static electricity that can accumulate on the surface of a polymer, reducing the risk of sparks. Previous studies have shown that curauá fibres may act as a reinforcing agent for thermoplastic matrices, such as polyethylene [3, 4], polypropylene [5] and polyamide-6 [6, 7]. There are numerous studies in the literature reporting the electrical properties of polyaniline emeraldine salt [8-10], as well as its application in blends [11, 12], but there are few studies about composites constituted of vegetal fibres and conducting polymers [12-15].

Polyamide 6 (PA-6) is one of the most important engineering polymers manufactured in large quantities, for various applications and in various forms, such as fibres, films, and plastics. PA-6 accounts for a majority of the commercial production and application of polyamide [16]. Composites of polyamide-6 (PA-6) reinforced with curaua fibres were studied for the first time by Santos et al. [6] aiming at glass fibre replacement. These authors reported that the reinforcement effect in PA-6 was obtained with $20 \mathrm{wt} \%$ of curauá fibres, if suitable processing equipment and conditions are used. The modification of the surface of curauá fibres with polyaniline nanoparticles was performed for the first time by Souza et al. [13], through the in situ preparation of the polyaniline in the presence of the curauá fibres. The incorporation of PAni on the fibres allowed 2500 times increase in the electrical conductivity of the fibres. Blends of PAni and PA-6 were also prepared by Schettini et al. [12] with the purpose of comparing the casting and the in situ polymerization methods. These authors reported that the conductivity values for the blends prepared by both methods are in the range of antistatic materials.

In the previous work it was demonstrated that polyaniline coated curauá fibres (CF-PAni) are efficient to promote conductivity in a composite with polyamide-6 prepared in a mini-extruder [7]. This study showed that the percolation threshold can be obtained with a very low content of PAni on 
the fibres (1-2 wt $\%$ ) and, with $12 \mathrm{wt} \%$ of PAni, the conductivity of a composite is quite similar to the conductivity of pure PAni.

Due to the proven efficiency of curauá fibres in promoting the reinforcement effect, more advantageous in relation to other vegetal fibres, and the proven ability of the polyaniline coated curauá fibres to promote good electrical properties in PA-6 composites, the aim of this study is to evaluate how polyaniline, and the polyaniline coated curauá fibres, affect the microstructural, morphological and thermal properties of PA-6 and pristine curauá fibres. The oxidation state of the PAni was judged from XPS measurements that allowed the determination of the percentage of amine, imine and charged nitrogens present in the conducting polymer. The choice of the amount of polyaniline on the curauá fibres $(12 \mathrm{wt} \%)$ was based on a previous work in which the optimal results for mechanical and electrical properties were obtained [7].

\section{Material and methods}

\subsection{Materials and reagents}

Polyamide-6 (Nylon - MAZMID C 380, Mazzaferro Ind. e Com. de Polímeros e Fibras), aniline (Vetec, 99\%), p-toluenesulfonic acid, p-TSA, (Merck, 99\%), ammonium persulfate (Synth, 98\%) and heptahydrated copper sulfate (Synth, $99 \%$ ) were used in this work. The curauá fibres were supplied by Embrapa-PA (Belém do Pará-Brazil), milled in a three knife rotary mill (Rone, NFA 1533), extracted with acetone in a Soxhlet apparatus for 48 hours and dried at room temperature. The extraction aims to eliminate part of the lignin and promote fibrillation of the microfibril bundles. The average fibre length was calculated by the "Image Pró-Plus" software for image treatment, with a sampling of 400 fibres. The fibre length varied from 3 to $7 \mathrm{~mm}$.

\subsection{Synthesis of polyaniline coated fibres on a pilot-plant scale}

The modification of the fibres with polyaniline doped with p-toluene sulfonic acid was done using a $10 \mathrm{~L}$ glass reactor with a cooling jacket and equipped with a mechanical stirrer. The procedure was adapted from a previous paper [17]: $300 \mathrm{~g}$ of milled fibres were added to an aqueous solution of p-TSA ( $\left.1 \mathrm{~mol} \mathrm{~L}^{-1}\right)$ and aniline $\left(0.2 \mathrm{~mol} \mathrm{~L}^{-1}\right)$. The resulting mixture was kept under constant stirring for 1 hour at room temperature. Thereafter, $2 \mathrm{~L}$ of an aqueous solution of $\left(\mathrm{NH}_{4}\right)_{2} \mathrm{~S}_{2} \mathrm{O}_{4}\left(0.3 \mathrm{~mol} \mathrm{~L}^{-1}\right)$ and p-TSA $\left(1 \mathrm{~mol} \mathrm{~L}^{-1}\right)$ was added dropwise and the mixture was kept under mechanical stirring at $-5^{\circ} \mathrm{C}$ by pumping a cooling solution through the reactor jacket. After this time the medium became green and the resulting mixture was removed from the reactor using a valve at its bottom, filtered and the modified fibres were washed with a 2:5 (v/v) water: ethanol solution.

\subsection{Formulations}

The amount of PAni coated on the fibres was calculated using the results provided by $\mathrm{CHN}$ (carbon, hydrogen and nitrogen) elemental analysis [7], with 
the content of PAni calculated as percentage of nitrogen in the CF-PAni after subtracting the percentage of nitrogen in pure curauá fibre. Then, this result was compared with the percentage of nitrogen in pure PAni. The evaluated PAni contents on the curauá fibres were $12 \mathrm{wt} \%$. PA-6/CF-PAni composites were prepared with $95 \mathrm{wt} \%$ of PA- 6 and $5 \mathrm{wt} \%$ of conducting curauá fibres.

\subsection{Processing conditions}

Before processing, the PA- 6 was dried in a vacuum oven at $120^{\circ} \mathrm{C}$ for $4 \mathrm{~h}$ and the modified fibres were dried in a vacuum oven at $60^{\circ} \mathrm{C}$ for $2 \mathrm{~h}$. Composites were processed in a co-rotating intermeshing twin-screw extruder (ZSK $26 \mathrm{Mc}$, Coperion Werner and Pfleiderer, Germany, $\mathrm{L} / \mathrm{D}=44)$. The used temperature profile (from feed to die) was $200-220^{\circ} \mathrm{C}$ with a screw rotation of $300 \mathrm{rpm}$. The PA-6 matrix was fed using a gravimetric dosimeter $(5 \mathrm{~kg} / \mathrm{h})$ connected to the main feeding funnel whilst the fibres were fed using the side-feeder at a rotation speed of $250 \mathrm{rpm}$. The processing time for $1 \mathrm{~kg}$ of material was $12 \mathrm{~min}$. Degassing was used during processing.

\subsection{X-ray diffraction}

In order to obtain the structural and microstructural information about the PA-6 composites and respective components (pristine curauá fibre, PAni coated curauá fibre and PA-6), X-ray diffraction measurements were carried out. The wide angle X-ray diffraction patterns were collected on a Bruker AXS D8-Focus diffratometer in reflection Bragg-Brentano geometry, using $\mathrm{Cu} \mathrm{K \alpha}$ (Ni filter) with $2 \Theta$ step of $0.02^{\circ}$ and acquisition time of $15 \mathrm{~s} / \mathrm{step}$. In order to calculate the degree of crystallinity of the initial and modified fibres, the diffraction patterns were decomposed on crystalline and amorphous components using TOPASAcademic software [18]. Two diffraction peak profiles were described by two independent pseudo Voigt functions. The amorphous component was modelled by a split pseudo Voigt function. All parameters of the fitting functions were allowed to vary.

\subsection{X-ray photoelectron spectroscopy (XPS)}

XPS analyses were performed in an ultra-high vacuum station (analysis chamber pressure of $10^{-10}$ mbar) using a dual anode X-ray source $(\mathrm{Al}=1486.7 \mathrm{~K} \alpha \mathrm{eV})$. A spectrum energy calibration was performed with respect to the carbon 1s peak (binding energy of $284.6 \mathrm{eV}$ ). PAni was analysed in the form of a film deposited by casting using the solvent NMP (n-dimethyl pyrrolidone). The polyaniline coated curauá fibres and PA-6 composites were analysed in pellet form (ca. $1.2 \mathrm{~cm}$ of diameter and $0.5 \mathrm{~mm}$ of thickness) prepared by applying a pressure of $1.2 \mathrm{MPa}$.

\subsection{Scanning electron microscopy (SEM)}

SEM images were collected in a Nova Nanolab 600 dual beam microscope at $5 \mathrm{keV}$ accelerating energy and current of $50 \mathrm{pA}$. 


\section{Results}

\subsection{X-ray diffraction (XRD)}

X-ray diffraction is routinely used for the determination of relative crystalline/amorphous phase content (degree of crystallinity) in a mixture of polymeric compounds $[19,20]$.

The basic approach to calculation of a degree of crystallinity from X-ray diffraction data is to find a ratio of an integrated intensity of crystalline phases to an integrated intensity of a whole sample. In a sample with completely random orientation of crystallites, the area under the peaks in a diffraction pattern is directly proportional to the integrated intensity [21]. Estimations of the relative crystalline/amorphous phase content was done with the help of eqn (1), in which $A_{a}$ is the area of diffraction peak related to an amorphous component and $A_{c}$ is the residual area below Bragg peaks of the crystalline phases.

$$
\mathrm{X}=\frac{A_{c}}{A_{c}+A_{a}} \cdot 100 \%
$$

The diffraction patterns collected from the samples of curauá fibres and from PAni coated curauá fibres are shown in fig. $1 \mathrm{a}$ )-b). Lower diffraction intensities and broader diffraction peaks are observed in the case of pure fibre sample. This can be explained primarily by a lower compaction density of fibres compared to PAni coated fibres in a sample holder. As a consequence, a smaller overall amount and an effectively thicker layer of a sample, which introduces higher geometrical aberrations, are probed by X-rays in the case of the pure fibre sample. Nevertheless, one can see an excellent agreement between the two diffraction patterns indicating the proximity of structural properties of the fibres. The main features in the diffraction patterns are similar to the ones observed in cellulosic fibres [22] and consistent with the $\mathrm{I}_{\beta}$ polymorph of a cellulose [23, 24]. A significant amorphous background is superimposed with diffraction peaks. In addition to the above features, weak diffraction peaks can be observed in the diffraction pattern of curauá fibres (fig. 1a)). These peaks may come from the $\mathrm{I}_{\alpha}$ polymorph of cellulose which converts to $I_{\beta}$ phase during the coating by PAni [24]. As one can see from table 1, the process of coating of fibres does not alter their degree of crystallinity.

The diffraction patterns of the initial PAni sample exhibited a mixture of ordered crystalline and amorphous phases (fig. 1b). The diffraction peaks of PAni were not observed after coating in the diffraction pattern of PAni coated fibre (fig. 1b)). The small amount of overall PAni phase relative to the fibre 
a)

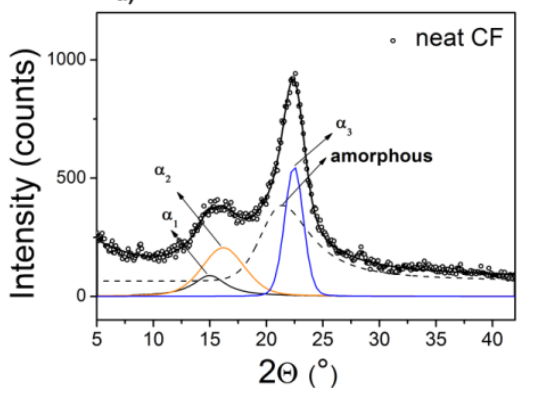

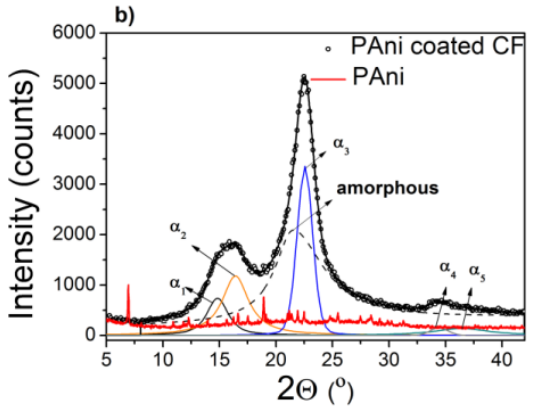

Figure 1: XRD pattern of fibres (circles) and profile decomposition on the amorphous (dashed line) and crystalline phases (solid lines), where a) pristine curauá fibres, and b) polyaniline coated curauá fibres and PAni sample synthesized without a substrate.

amount, and a conversion of the crystalline PAni phase to the amorphous one during the coating process can be responsible for this result. The transformation of crystalline PAni phase to an amorphous structure should reduce the electrical conductivity of PAni, since crystalline domains are considered the primary factors providing its high electrical conductivity [22, 23]. Indeed, the electrical measurements of PAni showed lower conductivity of the PAni phase on fibre than the initial PAni phase used for coating [7].

Table 1: Crystallographic data and fitting parameters obtained to the pristine curauá fibres and polyaniline coated curauá fibres.

\begin{tabular}{|c|c|c|c|c|c|c|c|c|}
\hline & \multicolumn{4}{|c|}{ Curauá fibres } & \multicolumn{4}{|c|}{ Polyaniline coated curauá } \\
fibres
\end{tabular}

\subsection{X-ray photoelectron spectroscopy (XPS)}

The doping ratio of PAni samples can be calculated by the $\mathrm{S} / \mathrm{N}$ (sulphur/nitrogen) ratio. XPS survey spectra, is able to measure, at the same time, nitrogen and sulfur content (only at the sample surface) through the peak 
area for each element, $\mathrm{N} 1 \mathrm{~s}(\sim 399 \mathrm{eV})$ and $\mathrm{S} 2 \mathrm{p}(\sim 168 \mathrm{eV})$, respectively, fig. $2 \mathrm{a})-\mathrm{c})$. The doping ratio $(\mathrm{S} / \mathrm{N})$ found by XPS analyses were 0.33 and 0.25 for PAni and CF-PAni, respectively. These values are in agreement with a 1:4 sulfur/nitrogen ratio, suggesting a good doping level.

Imine, amine and positively charged nitrogen atoms $\left(\mathrm{N}^{+}\right)$contents present in the PAni samples may be determined quantitatively using X-ray photoelectron spectroscopy by decomposition of the peak corresponding to $\mathrm{N} 1 \mathrm{~s}$ at 398.6 (imine, -N=), 399.3 (amine, -NH-) and 401.0 and $402.6 \mathrm{eV}$ (positively charged nitrogen, $-\mathrm{N}={ }^{+}$and $\left.-\mathrm{NH}^{+}{ }^{+}\right)[25]$.

$\mathrm{N}$ 1s peak of the polyaniline emeraldine base form should be decomposed into two Gaussian curves of the same area, and with binding energies of 399.3 and $398.6 \mathrm{eV}$, corresponding to the amine and imine groups, respectively [11]. However, after doping the polyaniline emeraldine base with protonic acids, as TSA, a reduction of the amine peak occurs and, simultaneously, the appearance of a shoulder in the region of higher binding energies (above $400 \mathrm{eV}$ ), corresponding to the positively charged nitrogen atoms. On the other hand, PAni in leucoemeraldine form (reduced form), should present only a single peak for the nitrogen 1s (amine), with a binding energy of approximately $399.4 \mathrm{eV}$. In fig. 2d), it can be observed that the PAni synthesized without a substrate presented $54 \%$ of aminic nitrogen, $26 \%$ of iminic nitrogen and approximately $20 \%$ of $\mathrm{N}^{+}$species. The low content of iminic nitrogens suggests a high doping level of PAni [13].

$\mathrm{N}$ 1s peaks of a CF-PAni sample consisted of the nitrogen peaks characteristic of PAni (imine, amine and positively charged nitrogens) and peaks related to a chemical bonding between PAni and cellulose components (fig. 2e)). The oxime linkage $(\mathrm{C}=\mathrm{N}-\mathrm{OH})$ appeared at $403.7 \mathrm{eV}$ and corresponds to hydrogen bonding between PAni and cellulose chains [26]. The peak at $404.6 \mathrm{eV}$ corresponds to nitrite groups (ONO), and the peak positioned at $406.5 \mathrm{eV}$ corresponds to nitrate groups. Qaiser et al. [27] reported that the disappearance of $\mathrm{NO}_{2}$ peaks in a
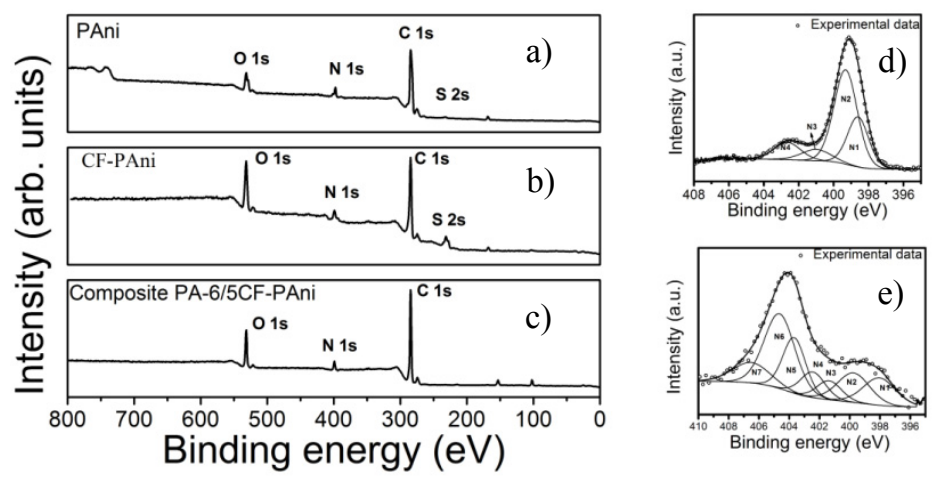

Figure 2: $\quad$ XPS survey spectra for: a) PAni and (b) CF-PAni, and c) composite 95PA-6/5CF-PAni; high resolution N 1s XPS spectra for d) PAni, and e) CF-PAni. 
cellulose ester microporous membrane covered with PAni shows the extent of coverage of the surface with PAni.

Analysing the results of table 2, it is interesting to observe that the PAni synthesized on the fibres surface is in the emeraldine salt form because the proportions of amine and iminic nitrogens are quite similar (12.3 and 13 at $\%$, respectively). The high content of positively charged nitrogens (15 at $\%$ ) shows the high doping level of the PAni on the fibres, that corroborates with the high conductivity results of this sample $\left(1.6 \mathrm{~S} \mathrm{~cm}^{-1}\right)$ [4].

Table 2: Summary of nitrogen species present in PAni and CF-PAni samples, as determined by XPS.

\begin{tabular}{|c|c|c|c|c|c|c|c|}
\hline \multicolumn{2}{|c|}{ Sample } & \multicolumn{3}{c|}{ PAni } & \multicolumn{3}{c|}{ CF-PAni } \\
\hline $\begin{array}{c}\text { N 1s } \\
\text { peak }\end{array}$ & Species & $\begin{array}{c}\text { Binding } \\
\text { energy } \\
(\mathrm{eV})\end{array}$ & FWHM & $\begin{array}{c}\text { At } \\
(\%)\end{array}$ & $\begin{array}{c}\text { Binding } \\
\text { energy } \\
(\mathrm{eV})\end{array}$ & FWHM & $\begin{array}{c}\text { At } \\
(\%)\end{array}$ \\
\hline N1 & $=$ N- & 398.6 & 1.4 & 26.0 & 398.0 & 2.5 & 12.3 \\
\hline N2 & -NH- $^{+}$ & 399.3 & 1.6 & 54.2 & 399.7 & 2.5 & 13.0 \\
\hline N3 & -N- $^{+}$ & 401.0 & 2.0 & 8.3 & 401.4 & 1.9 & 6.4 \\
\hline N4 & -NH- $^{+}$ & 402.6 & 1.8 & 11.5 & 402.4 & 1.9 & 8.6 \\
\hline N5 & - & - & - & - & 403.7 & 1.8 & 17.5 \\
\hline N6 & - & - & - & - & 404.6 & 2.4 & 31.6 \\
\hline N7 & - & - & - & - & 406.5 & 2.9 & 10.5 \\
\hline
\end{tabular}

\subsection{Scanning eletron microscopy (SEM)}

Polyaniline, in emeraldine salt form, exhibits the conductivity of the order of 1$400 \mathrm{~S} \mathrm{~cm}^{-1}$ [28]. Its structure is formed by crystalline/organized regions around amorphous regions [29]. The coexistence of these two phases can be observed in fig. 3a). This result is consistent with the high electrical conductivity values observed for the PAni synthesized in this work $\left(1.15 \mathrm{~S} \mathrm{~cm}^{-1}\right)$ [7].

In the micrography of PAni crystalline region (fig. 3b)), it is possible to observe PAni crystallites of a regular form with some crystallites at the nanometer scale (100-400 nm). This structural organization favours the hopping electrical conduction mechanism in which the carriers can tunnel from one crystallite to another through the amorphous regions. The micrography of the PAni supported on the curauá fibre shows higher amorphous contents than crystalline regions (fig. 3c)). This observation is consistent with the XRD pattern of the CF-PAni (fig. 1b)) where PAni crystalline peaks were not observed.

In the image of the 95PA-6/CF-PAni composite (fig. 3d)), an increase in the adhesion at the fibre-matrix interface is observed. SEM results are in agreement with the assumption that PAni is responsible for enhancing the fibre-matrix interaction through the formation of hydrogen bonds (previously shown by XPS results). The partial hydrolysis of PA-6 during extrusion produces carboxylic chain end groups that will interact with the amine groups of PAni by acid-base interactions, increasing the interfacial adhesion. 

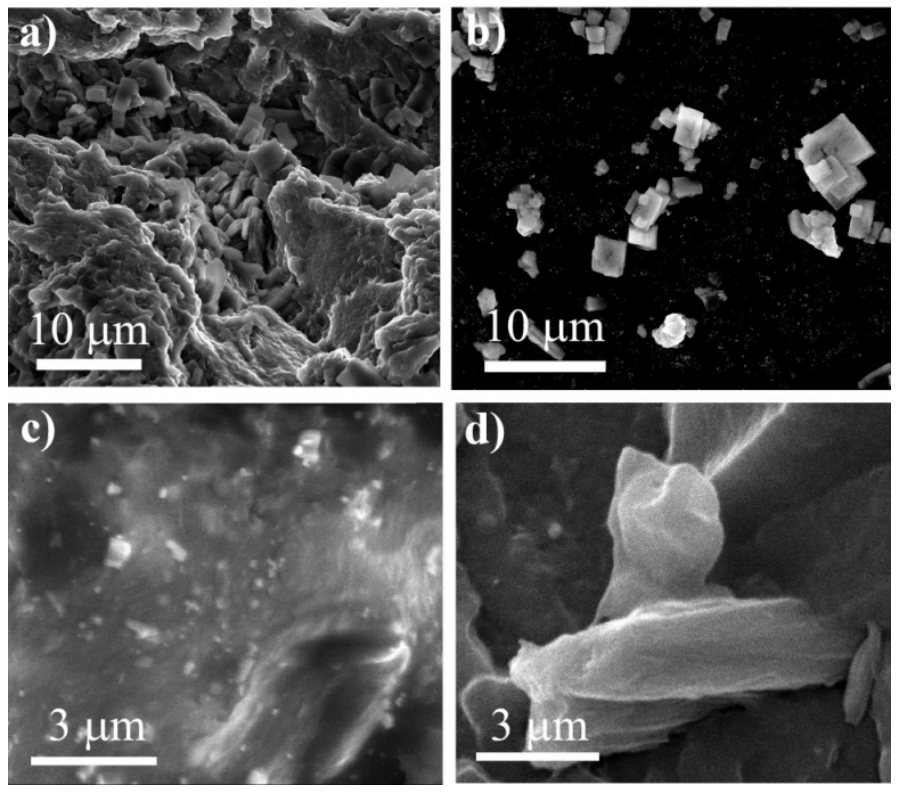

Figure 3: SEM images where: a) PAni doped with p-TSA showing amorphous and crystallines regions, b) PAni doped with p-TSA, showing only the crystalline region, c) polyaniline coated curauá fibre, and d) 95PA-6/5CFPAni composite.

\section{Conclusion}

PAni coatings of curauá surfaces are efficient ways to prepare composites with good electrical and mechanical properties, as already shown in a previous paper [7], without effluent generation and using large scale production (extrusion and injection moulded).

The X-ray diffraction combined with microscopy analyses of PAni synthesized without a substrate, showed crystalline domains embedded into amorphous regions in the sample, while the PAni synthesized on the fibres surface showed an apparent amorphization of PAni. XPS analysis showed that the PAni synthesized on the fibres was in emeraldine salt form, which corroborates with the good conductivity results observed for this sample [7]. The composite with 5 wt.\% of PAni presented good fibre-matrix adhesion caused by the hydrophilic character of all components (PAni, PA-6 and curauá fibres).

\section{References}

[1] Schettini, A.R.A., Peres R.C.D., Soares B.G., Synthesis of polyaniline/camphor sulfonic acid in formic acid medium and their blends 
with polyamide-6 by in situ polymerization. Synthetic Metals, 159, pp. 1491-1495, 2009.

[2] Hota P.R., Parida R.K., Das S.C., XRD and thermal characteristics studies of conducting polymers. Journal of Reinforced Plastics and Composites, 28, 2009, pp. 265-278.

[3] Araujo J.R., Waldman W.R., De Paoli M-A., Thermal properties of high density polyethylene reinforced with natural fibres: coupling agent effect. Polymer Degradation and Stability, 93, pp. 1770-1775, 2008.

[4] Araujo J.R., Mano B., Teixeira G.M., Spinacé M.A.S., De Paoli M-A., Biocomposites of high density polyethylene reinforced with curauá fibres: Mechanical, interfacial and morphological properties. Composites Science and Technology, 70, pp. 1637-1644, 2010.

[5] Spinacé M.A.S, Fermoselli K.K.G., De Paoli M-A., Recycled polypropylene reinforced with curauá fibres by extrusion. Journal of Applied Polymer Science, 112, pp. 3686-3694, 2009.

[6] Santos P.A., Spinacé M.A.S., Fermoselli K.K.G., De Paoli M-A., Polyamide-6/vegetal fibre composite prepared by extrusion and injection molding. Composite Part A: Applied Science and Manufacturing, 28, pp. 2404-2411, 2007.

[7] Araujo J.R., Adamo C.B., De Paoli M-A., Conductive composites of nylon-6 with polyaniline coated vegetal fibre. Chemical Engineering Journal, 174, pp. 425-431, 2011.

[8] Sengupta P.P., Kar P., Adhikari B., Influence of dopant in the synthesis, characteristics and ammonia sensing behavior of processable polyaniline. Thin Solid Films, 517, pp. 3770-3775, 2009.

[9] Mac Diarmid A.G. \& Epstein A.J., Polyanilines - A novel class of conducting polymers. Faraday Discussions of the Chemical Society, 88, pp. 317-332 1989.

[10] Dutta P., Biswas S., De S.K., Dieletric relaxation in polyaniline-polyvinyl alcohol composites. Materials Research Bulletin, 37, pp. 193-200, 2002.

[11] Rodrigues P.C., Muraro M., Garcia C.M., Souza G.P., Abbate M., Schreiner W.H., Gomes M.A.B., Polyaniline/lignine blends: thermal analysis and XPS. European Polymer Journal, 37, pp. 2217-2223, 2001.

[12] Schettini A.R.A., Peres R.C.D., Soares B.G., Synthesis of polyaniline/camphor sulfonic acid in formic acid medium and their blends with polyamide-6 by in situ polymerization. Synthetic Metals, 159, pp. 1491-1495, 2009.

[13] Souza Jr. F.G., Oliveira G.E., Rodrigues C.H.M., Soares B.G., Nele M., Pinto J.C., Natural Brazilian Amazonic (curauá) fibres modified with polyaniline nanoparticles. Macromolecular Materials and Engineering, 294, pp. 484-491, 2009.

[14] Chandran A.S., Narayanankutty S.K., An elastomeric conducting composite based on polyaniline coated nylon fibre and chloroprene rubber. European Polymer Journal, 44, pp. 2418-2429.

[15] Micusik M., Omastova M., Prokes J., Krupa I., Mechanical and electrical properties of composites based on thermoplastic matrices and conductive 
cellulose fibres. Journal of Applied Polymer Science, 101, pp. 133-142, 2006.

[16] Araujo J.R., Vallim M.R., Spinacé M.A.S., De Paoli M-A., Use of post consumer polyethylene in blends with polyamide-6: effect of the extrusion method and compatibilizer. Journal of Applied Polymer Science, 110, pp. 1310-1317, 2008.

[17] Araújo O.A. \& De Paoli M-A., Pilot plant scale preparation of dodecylbenzene sulfonic acid doped polyaniline in ethanol/water solution: control of doping, reduction of purification time and of residues. Synthetic Metals, 159, pp. 1968-1974, 2009.

[18] Coelho A.A. TOPAS - Academic Users Manual, 2005, http://members.optusnet.com.au/ alancoelho/

[19] Roe R.J., Methods of X-ray and neutron scattering in polymer science, Oxford University Press., New York, 2000.

[20] Silva V.P.R., Silva G.G., Caliman V., Rieumont J., Miranda-Pinto C.O.B., Archanjo B.S., Neves B.R.A., Morphology, crystalline structure and thermal properties of PEO/MEEP blends. European Polymer Journal, 43, pp. 3283-3291, 2007.

[21] Warren B.E., X-Ray Diffraction, Dover Publications: New York, 1990.

[22] Subramanian K., Kumar P., Jeyapal P. and Venkatesh N., Characterization of ligno-cellulosic seed fibre from wrightia tinctoria plant for textile applications - an exploratory investigation. European Polymer Journal, 41, pp. 853-861, 2005.

[23] Nishiyama Y., Langan P. and Chanzy H., Crystal structure and hydrogenbonding system in cellulose $\mathrm{I}_{\alpha}$ from synchrotron $\mathrm{X}$-ray and neutron fibre diffraction. Journal of American Chemical Society, 124, pp. 9074-9082, 2002.

[24] Kovalenko V.I., Crystalline Cellulose: structure and hydrogen bonds. Russian Chemical Reviews, 79, pp. 231-241, 2010.

[25] Li Z.F., Kang E.T., Neoh K.G., Tan K.L., Effect of thermal processing conditions on the intrinsic oxidation states and mechanical properties of polyaniline films. Synthetic Metals, 87, pp. 45-52, 1997.

[26] Pan W., Yang S.L., Li G., Jiang J.M., Electrical and structural analysis of conductive polyaniline/polyacrylonitrile composites. European Polymer Journal, 41, pp. 2127-2133, 2005.

[27] Qaiser A.A., Hyland M.M., Patterson D.A., Effects of various polymerization techniques on PAni deposition at the surface of cellulose ester microporous membranes: XPS and electrical conductivity studies. Synthetic Metals, 162, 958-967, 2012.

[28] Chance R., Bordeaux D., Brédas J.L., Silbey R., Handbook of conducting polymers, vol. 2, ed. Marcel Dekker Inc.: New York, pp. 825, 1986.

[29] Varma S.J., Xavier F., Varghese S., Jayalekshmi S., Synthesis and studies of exceptionally crystalline polyaniline thin films. Polymer International, 61, pp. 743-748, 2012. 